\title{
Estimating the Efficacy of a Commercial Phase I Inactivated Vaccine in Decreasing the Prevalence of Coxiella burnetii Infection and Shedding in Red Deer (Cervus elaphus)
}

\author{
David González-Barrio ${ }^{1}$, José Antonio Ortiz ${ }^{2}$ and Francisco Ruiz-Fons ${ }^{1 *}$
}

${ }^{1}$ Health and Biotechnology (SaBio) Group, Instituto de Investigación en Recursos Cinegéticos IREC (CSIC-UCLM-JCCM), Ciudad Real, Spain, ${ }^{2}$ Medianilla Red Deer Genetics, Benalup-Casas Viejas, Spain

The red deer (Cervus elaphus) is a relevant reservoir for Coxiella burnetii in Iberia. C. bur-

\section{OPEN ACCESS}

Edited by:

James Allen Byrd,

Agricultural Research Service-

United States Department of

Agriculture (ARS-USDA),

United States

Reviewed by:

Rafael Antonio Casarin Penha Filho, Universidade Estadual Paulista Júlio de Mesquita Filho (UNESP), Brazil

Carol Geralyn Chitko-McKown,

US Meat Animal Research Center, United States

*Correspondence:

Francisco Ruiz-Fons josefrancisco.ruiz@uclm.es

Specialty section:

This article was submitted to Veterinary Infectious Diseases,

a section of the journal

Frontiers in Veterinary Science

Received: 25 August 2017 Accepted: 21 November 2017 Published: 06 December 2017

Citation:

González-Barrio D, Ortiz JA and Ruiz-Fons $F$ (2017) Estimating the Efficacy of a Commercial Phase I Inactivated Vaccine in Decreasing the Prevalence of Coxiella burneti Infection and Shedding in

Red Deer (Cervus elaphus).

Front. Vet. Sci. 4:208

doi: 10.3389/fvets.2017.00208 netii genotypes that infect red deer also infect humans and domestic animals. Integrated control approaches that target both domestic and wild ruminants are, therefore, required to reduce $C$. burnetii infection risks in Iberia, especially in wildlife-livestock-human interaction scenarios. The aim of this field experiment was to test the efficacy of an inactivated phase I vaccine [Inactivated phase I vaccine (IPIV); Coxevac ${ }^{\circledR}$ ] when used to control C. burnetii shedding prevalence and burden in red deer as a tool to prevent transmission to livestock and humans. A semi-extensively bred red deer population in which $C$. burnetii is endemic was used as a model of the lberian context. Around $75 \%$ of the reproductive hinds ( $>1$ year old; $N=441$ ) in the population were first vaccinated early in 2012 and were then revaccinated 3 weeks later; they were subsequently revaccinated biannually until January 2014. 75\% of the yearling females left as replacement in 2012 and 2013 were vaccinated in June and revaccinated thereafter following the same protocol. $25 \%$ of the population, including the replacement females, was kept as a control group throughout the study. Changes in the humoral immune response after vaccination were estimated by analyzing sera collected at 10 different times between January 2011 and January 2015. The vaccinated and control hinds were surveyed at $2.5,3.5$, and 4.5 months after calving in 2012, 2013, and 2014 to collect vaginal swabs, milk, and feces. The presence and burden of $C$. burnetii DNA in swabs, milk, and feces was evaluated by means of real-time PCR. Vaccination induced high antibody prevalence and levels. The proportion of animals shedding $C$. burnetii in vaginal secretions and milk did not change over time in the vaccination group with respect to the control group. In contrast, there was a significant reduction in the proportion of deer shedding $C$. burnetii in feces in both the vaccinated and control groups. The decrease in the proportion of fecal shedders coincided with a significant reduction in the incidence of infection of non-vaccinated yearling females in the population. This finding suggests that long-term vaccination with IPIV could reduce environmental contamination with $C$. burnetii and control transmission, perhaps making this a promising tool with which to control $C$. burnetii in red deer in the future.

Keywords: control, inactivated phase I vaccine, Q fever, wildlife, zoonosis 


\section{INTRODUCTION}

Q fever is a zoonotic disease caused by Coxiella burnetii, a Gramnegative bacterium, that infects animals and humans worldwide, causing a high economic impact (1-3); e.g., the total cost of the massive Q fever outbreak in the Netherlands between 2007 and 2010 was estimated as being $€ 600$ million $(4,5)$.

Clinical signs of $\mathrm{Q}$ fever in domestic and wild ruminants include returns to estrus, abortions, premature deliveries, stillbirths, and the birth of weak offspring with reduced survival rates $(2,6)$. It is estimated that $Q$ fever causes around $9 \%$ of the abortions in sheep flocks (7), which makes $\mathrm{Q}$ fever an important disease in domestic ruminant production. In wildlife, $\mathrm{Q}$ fever is a major threat to the success of controlled breeding programs for endangered wild ruminants, e.g., the Saharawi dorcas gazelle (Gazella dorcas neglecta) breeding program (Abaigar T., personal communication) and the productivity of farmed wild ungulates $(8,9)$. C. burnetii replicates efficiently in the placenta of ruminants and is subsequently shed in vaginal secretions, milk, and feces by infected females (10). Massive shedding occurs mainly around parturition or after reproductive failure and leads to high concentrations of infective C. burnetii in the environment that favors transmission through contaminated aerosols.

Domestic ruminants are the main reservoirs of $C$. burnetii for humans (1). However, C. burnetii is a multi-host pathogen with a wide host range (11), and like other multi-host pathogens $(12,13)$, its transmission at the wildlife-livestock-human interface may have increased with the demographic changes undergone by particular wild species, e.g., ungulates (14). A recent large-scale study proved that the red deer (Cervus elaphus) is an important wild reservoir of C. burnetii in Iberia (15); 50\% of Iberian red deer populations are infected with C. burnetii, with an average individual antibody prevalence of $12.2 \%$, which is similar to values reported in domestic ruminant herds (16). Furthermore, red deer females shed C. burnetii after infection (9), and specific red deer genotypes infect humans (17). The current increasing demographic trends of red deer populations in Europe (14) would, therefore, ease C. burnetii transmission at the red deerlivestock-human interface. Preparedness to fight against $\mathrm{Q}$ fever would require integrated approaches targeting both domestic and wild reservoirs. To achieve this, it is necessary to improve current control strategies in domestic ruminants and develop new strategies with which to control the infection in key wild reservoirs in wildlife-livestock-human interaction scenarios.

Q fever control approaches seek to reduce the proportion of C. burnetii shedders as a means of reducing environmental contamination and transmission. The most efficient strategy by which to reduce the percentage of shedders in a herd is currently vaccination with inactivated phase I vaccines (IPIVs) (18). The use of IPIV in domestic ruminants reduces the risk of infection of naive animals (19-22). In endemic herds, vaccination with IPIV is less efficient in the short-term, and therefore, long-term vaccination is recommended $(19,23)$. In this context, this study aims to test the efficiency of the long-term use of commercial IPIV as regards reducing C. burnetii shedding in red deer. The efficacy of IPIVs in wild ruminants has not been tested to date; however, epidemiological and clinical studies suggest that $C$. burnetii infection is similar in wild and domestic ruminants $(6,8,9,11)$, and that basic knowledge acquired in domestic ruminants can be applied to wild ruminants. We seek to test the efficiency of IPIV in endemic scenarios because C. burnetii infects over $50 \%$ of Iberian red deer populations; any laboratory-controlled experimental approach carried out on red deer would most probably mimic what has been previously reported in domestic ruminants. Therefore, we carried out a field longitudinal vaccination experiment and monitored $C$. burnetii shedding prevalence and burden over 3 years using a semi-extensively bred deer population as a model.

\section{MATERIALS AND METHODS}

\section{The Study Population}

The experiment was performed on a semi-extensively bred red deer population located in the province of Cádiz in southern Spain. The deer are semi-extensively bred in a forest-shrubprairie habitat divided into different plots by high-wire fencing. The animals are kept in separate batches according to their sex and productive status. The number of deer in the population is around 500 hinds and 100 stags. They are kept within large fenced (6-8 ha) enclosures in batches of 60-80 reproductive females; the males are kept in separate enclosures. The animals are identified with individual ear tags.

A strict management protocol is implemented in the study population so as to minimize the stress associated with handling, especially during critical stages of the production cycle, such as calving. Although bred in controlled conditions, these deer are not domestic animals and consequently get highly stressed when they are restrained for sample collection. The reproductive females ( $>1$ year old) give birth to calves by the end of April. The calves remain with their mothers in enclosures until they are weaned at around 3.5 months of age, after which they are split into male and female batches separated from the hinds and stags. All the calves are handled for routine pathogen monitoring at the age of $2.5,3.5$, and 7 months. The reproductive females are handled annually in winter (January-February) and two/three times in summer (July, August, and September). A selection of 13-monthold yearling females is left annually as replacement, and these are randomly allocated to existing batches of reproductive hinds. The strict handling protocol precluded the design of a more accurate monitoring protocol for the vaccination experiment. Nonetheless, this was still a unique opportunity to test the efficacy of IPIVs in an endemic red deer population, something that is extremely difficult to achieve in a free-roaming deer population. Furthermore, very few attempts to monitor the efficiency of IPIVs in real endemic scenarios have been performed to date and none with wild ruminants.

\section{Dynamics of C. burnetii Infection in the Study Population}

The long-term monitoring of $C$. burnetii in the study population confirmed that it is endemic $(9,24,25)$. Transmission takes place mainly around the calving season (25). Clinical reproductive signs associated with infection with C. burnetii have never been 
observed directly in this population because the hinds remain undisturbed in enclosures during the last third of the gestation period and around calving. However, C. burnetii infection has been associated with increased reproductive failure in the study population (9) and in other deer populations (26).

European wild rabbits (Oryctolagus cuniculus) are abundant in the study area and share the habitat with the deer. Rabbits are also true C. burnetii reservoirs (27). However, the interference of rabbits in the dynamics of $C$. burnetii infection in deer is expected to be low because distinct $C$. burnetii genotypes infect the deer and rabbits on the study site $(17,28)$.

\section{Design of the Experimental Vaccination Trial} An IPIV that has been widely tested on European domestic ruminants was selected for the experiment $\left(\right.$ Coxevac $^{\circledR}$, CEVA Santè Animale, France). Coxevac is commercially available in Spain, and its use in mammal species is approved by the European Medicines Agency. The experiment was designed in compliance with the recommendations of the vaccine manufacturer. The experiment was approved by the Research Ethics Commission of the Animal Ethics Committee of Castilla_La Mancha University.

The experiment targeted hinds with a history of natural exposure to C. burnetii as a means of estimating the usefulness of IPIV as regards reducing the risk of infection in free-ranging infected red deer populations. Previous vaccination experiments carried out on domestic ruminants suggest revaccination every 9-12 months $(29,30)$. However, long-term series serological data of the study population showed that the average life of antibodies produced after natural infection was around 5-6 months (25). This finding suggested that protection linked to humoral immunity would be boosted with revaccination every 6 months.

In January 2012, the first dose of the vaccine was given to 320 of the 441 reproductive hinds comprising the herd $(72.6 \%)$, while the rest $(n=121,27.4 \%)$ were left as a control group (2010 cohort). We allocated vaccinated and control animals to each existing batch on the farm to evaluate the effect of vaccination on coexisting non-vaccinated mates. Any female batch on the farm, therefore, contained both vaccinated and non-vaccinated animals. Vaccinated animals were revaccinated 3 weeks after vaccination (29). Thereafter, the animals in the vaccinated group were revaccinated biannually until January 2014. In June 2012, 93 of the 124 (75.0\%) yearling females born in 2011 that were kept as replacement (2011 cohort) were vaccinated. Revaccination was performed 3 weeks later and biannually (Figure 1); 31 animals (25.0\%) were left unvaccinated. In June 2013, 104 of the 134 hinds $(77.6 \%)$ of the cohort of replacement females born in 2012 were vaccinated and were revaccinated 3 weeks later and biannually thereafter; 30 females $(22.4 \%)$ were left unvaccinated. The proportion of hinds in the vaccination vs. the control group remained at 3:1 throughout the experiment. The vaccine was injected subcutaneously ( $3 \mathrm{ml}$ dose) with an automatic injector (Serena 5TPFS, Pimex, Spain). A descriptive summary of the vaccination protocol applied to each cohort is provided in Figure 1.

\section{Monitoring of $\boldsymbol{C}$. burnetii Shedding}

In domestic ruminants, Coxevac has shown the potential to reduce the prevalence of shedders and the burden of $C$. burnetii shed $(18,22,29)$. In this study, the efficacy of the vaccination was evaluated by collecting vaginal secretions, milk, and feces at different times-2.5 (July), 3.5 (August), and 4.5 (September) months-after calving in 2012, 2013, and 2014 (Table 1; Figure 1). Samples were collected from a random subset of hinds from both the vaccinated and the control groups whenever they were sampled. Vaginal secretions were collected using sterile cotton swabs. The milk was extracted by hand into sterile tubes after disinfecting the deer's nipples with $1 \%$ chlorhexidine and discarding the first three milk shots. Feces were collected directly from the rectum using sterile disposable latex gloves. The vaginal swabs, milk, and feces were transported to the laboratory in a state of refrigeration and preserved frozen at $-20^{\circ} \mathrm{C}$ until their analysis. The researchers took appropriate biosecurity measures during sample collection, transportation, and analysis.
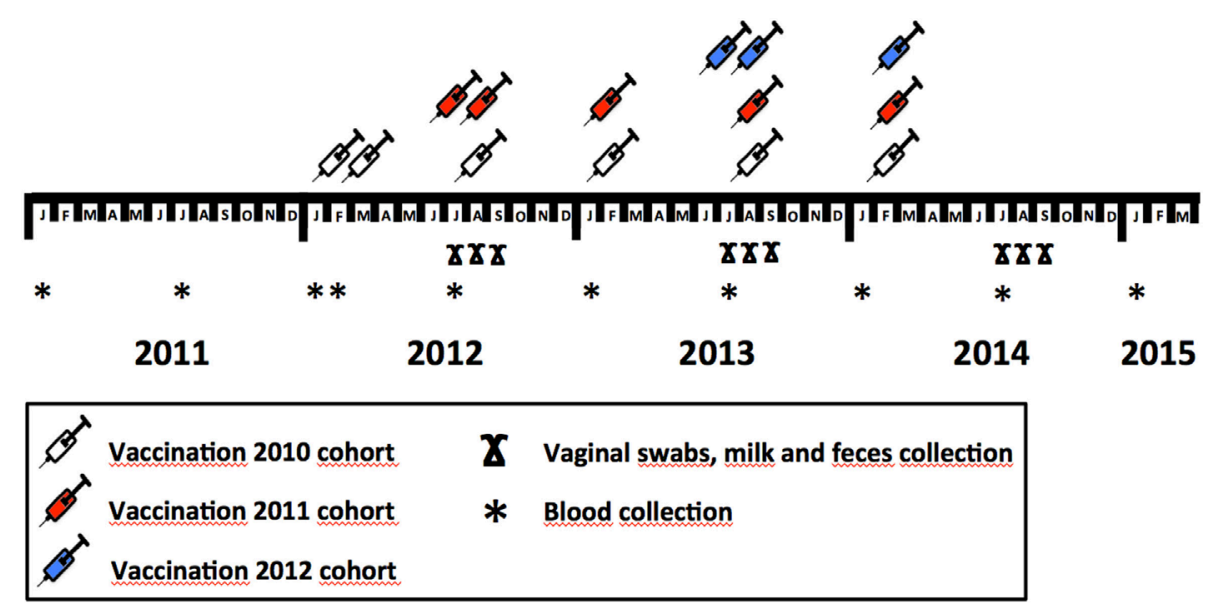

FIGURE 1 | Vaccination and sample collection schedule throughout the study, showing the protocol employed to implement vaccine in different deer cohorts (2010, 2011, and 2012) and sample-blood, vaginal swabs, milk, and feces - collection months within study years. 
TABLE 1 | Sample size throughout sample type and sampling time according to the allocation of animals to vaccinated (Vacc) and control (Cont) groups.

\begin{tabular}{|c|c|c|c|c|c|c|c|}
\hline \multirow[t]{2}{*}{ Sample type } & \multirow[t]{2}{*}{ Group } & \multirow[t]{2}{*}{ Months } & \multicolumn{5}{|c|}{ Year } \\
\hline & & & 2011 & 2012 & 2013 & 2014 & 2015 \\
\hline \multirow[t]{7}{*}{ Vaginal swab } & Vacc & July & & 45 & 40 & 62 & \\
\hline & & August & & 17 & 41 & 41 & \\
\hline & & September & & 13 & 28 & 26 & \\
\hline & Cont & July & & 25 & 12 & 19 & \\
\hline & & August & & 14 & 10 & 19 & \\
\hline & & September & & 11 & 16 & 5 & \\
\hline & Subtotal & & & 125 & 147 & 172 & \\
\hline \multirow[t]{7}{*}{ Milk } & Vacc & July & & 17 & 26 & 16 & \\
\hline & & August & & 17 & 12 & 34 & \\
\hline & & September & & 18 & 31 & 20 & \\
\hline & Cont & July & & 12 & 7 & 6 & \\
\hline & & August & & 13 & 2 & 12 & \\
\hline & & September & & 9 & 17 & 3 & \\
\hline & Subtotal & & & 86 & 95 & 91 & \\
\hline \multirow[t]{7}{*}{ Feces } & Vacc & July & & 13 & 33 & 28 & \\
\hline & & August & & 7 & 36 & 35 & \\
\hline & & September & & 12 & 35 & 25 & \\
\hline & Cont & July & & 7 & 11 & 9 & \\
\hline & & August & & 9 & 12 & 11 & \\
\hline & & September & & 12 & 16 & 5 & \\
\hline & Subtotal & & & 60 & 143 & 113 & \\
\hline \multirow[t]{7}{*}{ Serum } & Vacc & January & 48 & 313 & 77 & 89 & 72 \\
\hline & & February & & 313 & & & \\
\hline & & July & 80 & 121 & 124 & 87 & \\
\hline & Cont & January & 26 & 120 & 50 & 60 & 57 \\
\hline & & February & & 120 & & & \\
\hline & & July & 43 & 81 & 89 & 58 & \\
\hline & Subtotal & & 197 & 1068 & 340 & 294 & 129 \\
\hline
\end{tabular}

Moreover, to estimate the presence of $C$. burnetii antibodies in the vaccinated and control hinds before and after vaccination, blood was collected from the jugular vein and placed in sterile 10-ml tubes without an anticoagulant (Table 1; Figure 1). This blood was transported to the laboratory at $4^{\circ} \mathrm{C}$, centrifuged at $3,000 \mathrm{~g}$ for $10 \mathrm{~min}$, and the serum obtained was preserved at $-20^{\circ} \mathrm{C}$ until the analyses were performed. Blood samples were collected at 10 different times between 2010 and 2015 (Figure 2).

\section{Molecular Analyses}

The DNA from vaginal swabs, milk, and feces was extracted using a commercial DNA extraction and purification kit (DNeasy Blood \& Tissue kit, Qiagen, Germany) following the protocols provided by the manufacturer. DNA extraction from swabs was optimized by keeping the swabs at $56^{\circ} \mathrm{C}$ for $30 \mathrm{~min}$ in a solution containing $20 \mu \mathrm{l}$ of proteinase $\mathrm{K}$ and $200 \mu \mathrm{l}$ of AL buffer. The swabs were subsequently vortexed vigorously for $15 \mathrm{~s}$, removed from the tubes, and discarded. The remaining sample was kept for 30 additional minutes at $56^{\circ} \mathrm{C}$, after which the manufacturer's blood extraction protocol was followed. Each sample of milk $(200 \mu \mathrm{l})$ was mixed directly with ATL and proteinase $\mathrm{K}$ and incubated for $3 \mathrm{~h}$ at $56^{\circ} \mathrm{C}$, and the manufacturer's blood extraction protocol was then followed. One gram of each fecal sample was mixed with $4 \mathrm{ml}$ of
TE buffer (Tris Base $10 \mathrm{mM}$, EDTA $1 \mathrm{mM}, \mathrm{pH}$ 8), vortexed for $30 \mathrm{~s}$, and centrifuged at 3,000 $\mathrm{g}$ for $2 \mathrm{~min}$. Thereafter, $200 \mu \mathrm{l}$ of the supernatant were treated with proteinase $\mathrm{K}(20 \mu \mathrm{l})$ and ATL buffer $(180 \mu \mathrm{l})$ for $30 \mathrm{~min}$ at $70^{\circ} \mathrm{C}$, and the extraction was completed following the manufacturer's blood and tissue extraction protocol. The concentration of DNA in each aliquot was quantified (NanoDrop 2000, Thermo Scientific, Waltham, MA, USA), and the aliquots were frozen at $-20^{\circ} \mathrm{C}$ until the PCR was performed. To prevent and detect sample cross-contamination, negative controls (Nuclease free water; Promega, Madison, WI, USA) were included in every 10 samples during the DNA extraction procedure. The DNA samples were analyzed by means of a realtime PCR (qPCR), targeting a transposon-like repetitive region of C. burnetii as described previously $(15,31)$. SsoAdvanced ${ }^{\mathrm{TM}}$ Universal Probes Supermix (BioRad, USA) was used in qPCR according to the manufacture's specifications. DNA extraction and PCR were performed in separate laboratories under biosafety level II conditions (BIO II A Cabinet, Telstar, Spain) to avoid cross-contamination. DNA from Coxevac was used as a qPCR positive control. Samples were considered positive to the presence of C. burnetii DNA at a cycle threshold (Ct) below 40.0 (31).

\section{Serological Analyses}

The presence of specific antibodies against C. burnetii in deer sera was determined by using a commercial indirect ELISA test (PrioCHECK ${ }^{\mathrm{TM}}$ Ruminant Q Fever Ab Plate Kit, Thermo Fisher Scientific, USA), as previously reported (15). The ELISA results were expressed as the sample-to-positive control ratio (SP). For each sample, the SP was calculated according to the formula:

$$
\mathrm{SP}=\frac{(\mathrm{ODs}-\mathrm{ODnc})}{(\mathrm{ODpc}-\mathrm{ODnc})} \times 100,
$$

where ODs is the optical density of the sample at a dual wavelength of 450-620 nm, ODnc is the optical density of the negative control, and ODpc is the optical density of the positive control. All SP values $\leq 40$ were considered negative, whereas $\mathrm{S} / \mathrm{P}$ values $>40$ were considered positive. The SP ratio was considered as a proxy of the level of antibodies against C. burnetii, as suggested by the manufacturer.

\section{Statistical Analyses}

Statistical analyses were carried out to compare the prevalence and shedding burden of C. burnetii in vaginal swabs, milk, and feces in vaccinated vs. control groups to test the hypothesis that deer immunized with Coxevac would undergo a reduction in C. burnetii shedding. The humoral immune response to vaccination in comparison to the control animals was evaluated by comparing seroprevalence and average antibody levels.

Chi-square tests were employed to compare C. burnetii DNA prevalence in vaginal swabs, milk, and feces, and antibody prevalence between the vaccinated and control groups at each sampling time. Mann-Whitney $U$ non-parametric tests were run to compare the burden of shed C. burnetii (average $\mathrm{Ct}$ ) in vaginal swabs, milk, and feces and antibody levels (average SP) in serum between the vaccinated and control groups. Finally, to assess the effect of time on C. burnetii shedding and to attain the evolution 

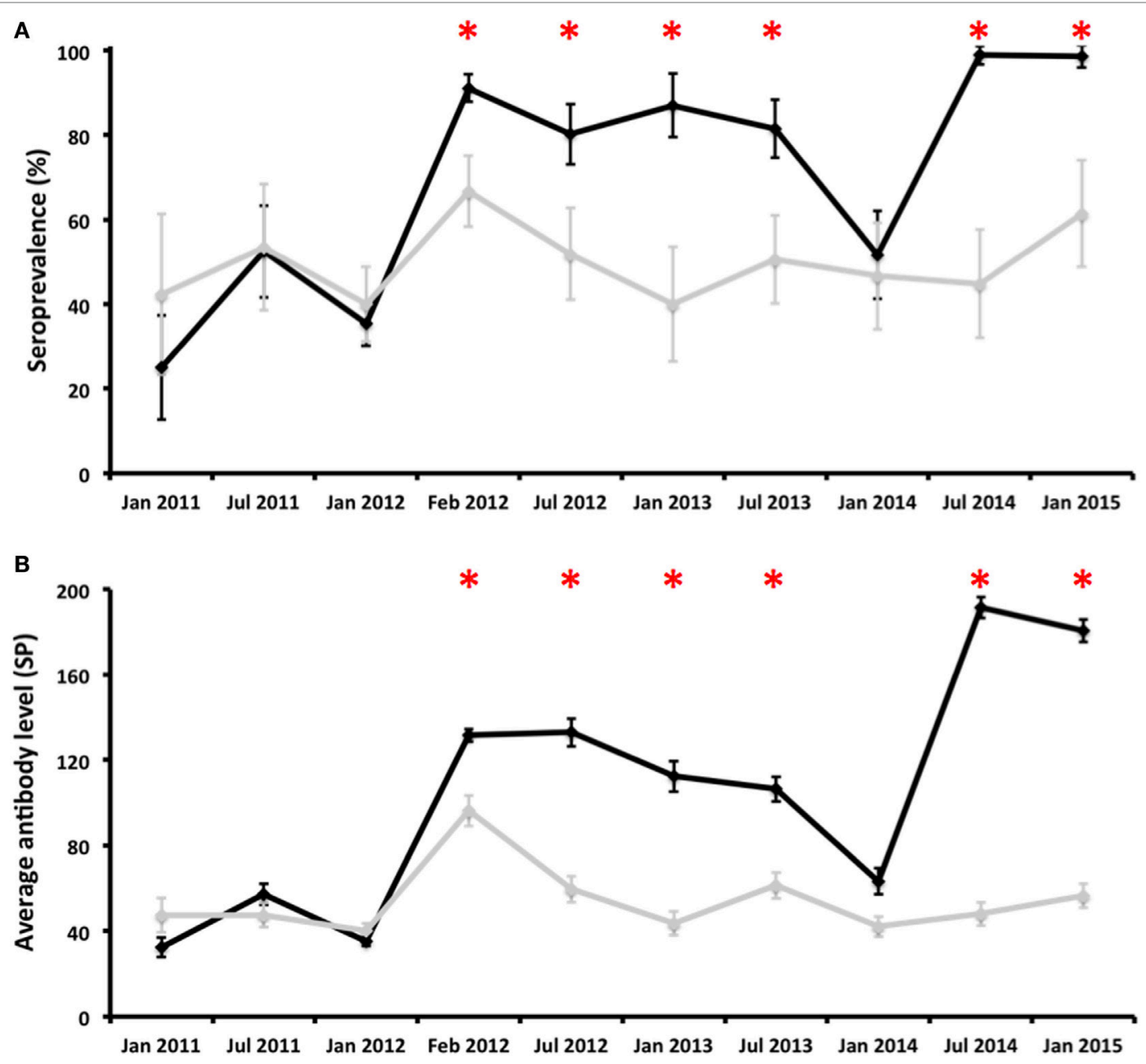

FIGURE 2 | Evolution of the proportion of antibody-positive deer [seroprevalence (\%); (A)] and average antibody levels [SP; (B)] in vaccinated (black line) and control (gray line) groups throughout the experiment. Error bars represent 95\% confidence intervals estimated for SER and the estimated SD from the mean for average antibody levels. *Statistically significant differences $(\rho<0.05)$ between vaccinated and control groups on a particular sampling date.

of the level of antibodies in vaccinated and control groups, we performed Spearman correlations.

Statistical analyses were run using IBMS SPSS v22.0 software (IBM, Armonk, New York, NY, USA). Ninety-five percent confidence intervals ( $95 \%$ CIs) were estimated for prevalence values according to the expression $95 \% \mathrm{CI}=1.96[p(1-p) / n]^{1 / 2}$, where " $p$ " is the prevalence in a unitary value and " $n$ " is the size of the sample employed to estimate the prevalence.

\section{RESULTS}

\section{Humoral Immune Response after Vaccination}

Two hundred and two hinds that were vaccinated in January 2012 (2010 cohort) were ELISA negative before vaccination. Three weeks after vaccination, 187 of them (92.6\%) were ELISA positive. Five months after revaccination, 170 of them were re-analyzed by means of ELISA, and all of them (100.0\%) were positive. The effect of vaccination on the humoral response was also evidenced by the increase in the average level of antibodies; seronegative hinds had an average SP value of 14.4 right before vaccination that increased after vaccination (132.1) and remained similar after revaccination (121.9). In contrast, hinds from the control group that were ELISA negative before the start of the experiment had average SP values of 14.9 in January 2012, 86.5 in February 2012, and 65.7 in July 2012. 90.0\% (9/10) of the deer from the 2011 cohort that were ELISA negative before vaccination were seropositive 3 weeks later. The level of antibodies underwent a fivefold increase from June 2012 (21.7) to July 2012 (99.1) in the vaccinated group; the average SP values in the control group remained similar throughout this period: 19.8 and 21.7, respectively. The change in the presence and level of antibodies after vaccination could not be estimated for the 2012 cohort because none of the seronegative individuals surveyed right before vaccination (June 2013) was surveyed in July 2013. 
Sera $(N=2028)$ were obtained from experimental hinds at 10 different times from January 2011 to January 2015 (Table 1). Antibody prevalence remained above the $80 \%$ in the vaccination group after the first vaccination-with the exception of January 2014 - and above that observed in the control group (Figure 2). Differences in antibody prevalence between the vaccination and control groups were statistically significant at different times after vaccination (Figure 2). The pattern observed in the average antibody level was similar (Figure 2). Indeed, the vaccinated animals had average SP values close to 200, an unusual SP value in naturally infected ruminants (16). Differences in antibody levels between the vaccinated and control hinds after vaccination were always statistically significant, except in January 2014 (Figure 2). The seroprevalence and average antibody levels had statistically significantly positive time trends in the group of vaccinated animals (rho $=0.697, p<0.05$ and rho $=0.745, p<0.05$, respectively); neither the seroprevalence nor the antibody level in the control group had statistically significant time trends (Figure 2).

\section{Patterns of C. burnetii Shedding after Vaccination}

A total of 444 vaginal swabs, 272 milk samples and 316 fecal samples from 319 hinds (228 vaccinated and 91 controls) were investigated in 2012, 2013, and 2014 (Table 1). The shedding patterns of $C$. burnetii in vaginal secretions, milk, and feces were analyzed separately.

Coxiella burnetii DNA was detected in vaginal swabs, milk, and feces during any of the surveys carried out after calving, that is, until almost the fifth month after calving (Figure 3). Almost no statistically significant differences in shedding prevalence and in average qPCR Ct values were observed between the vaccinated and control animals (Figures 3 and 4). In general terms, the shedding prevalence in vaginal swabs, milk, and feces (Figure 3), although not the burden of shed bacteria (Figure 4), tended to decrease from the time of calving in any of the three years surveyed and in both the vaccination and control groups. Interestingly, shedding in milk seems to be more limited as regards the time after calving than shedding through vaginal mucus and feces (Figure 3). Another interesting observation was the absence of vaginal shedding in September 2014 and in both the vaccinated and control groups, which contrasts with previous years.

The shedding prevalence of $C$. burnetii in feces, but not the burden of shed bacteria, had a statistically significant decreasing time trend in both the vaccinated (rho $=-0,850, p<0.01$ ) and control (rho $=-0.870, p<0.01$ ) groups (Figure 3). In 2012, the average shedding prevalence was over $50 \%$, in contrast to 2014 in which it was below $20 \%$, when none of the surveyed animals belonging to the control group shed C. burnetii through feces.

\section{DISCUSSION}

Inactivated phase I vaccines have been used in domestic ruminants as a tool to reduce C. burnetii shedding by infected animals to limit environmental contamination and thus decrease the risk of infection for naive animals and humans $(18,19,21)$. For an optimal vaccine efficacy, populations may fulfill one common pre-requisite, $C$. burnetii shall not be present in the population or it shall only circulate at low prevalence $(19,20)$. However, since C. burnetii is widespread in domestic and wild ruminant populations almost worldwide, there have been attempts to estimate the accuracy of IPIV in controlling infection in endemic scenarios. Some of these studies suggest that IPIV might be useful to control C. burnetii infection if long-term vaccination is performed (21). Therefore, and since C. burnetii infects around 50\% of red deer populations in the Iberian Peninsula (15), we opted to design a long-time vaccination trial. For the first time in scientific literature, this field experiment provides evidence that IPIV may be effective in reducing the shedding of C. burnetii by red deer if applied on a long-term basis; additionally, it opens a line of research on C. burnetii control in wildlife. The chances of delivering vaccines to wildlife to control relevant pathogens, e.g., rabies virus, classical swine fever virus, or animal tuberculosis (32-34), are increasing because of the progress made in research on efficient oral vaccines and vaccine delivery methods to wildlife (35). Vaccinating wildlife to control pathogens is not, therefore, a utopia. If this to be made possible, it is important to evaluate potential approaches in wildlife, especially with regard to pathogens that are shared with domestic animals and humans.

\section{Methodological Considerations}

This study arose from the need to study strategies with which to control C. burnetii infection in a semi-extensively bred red deer population in which $\mathrm{Q}$ fever was associated with increased reproductive disorders (9). We designed the experiment by assuming that the basic effects of IPIV would not significantly differ in domestic and wild ruminants. We considered that designing a laboratory-controlled vaccination-challenge experiment for red deer would be (i) unaffordable owing to the logistical constraints and the cost of keeping red deer in BSL3 facilities for a long time period; (ii) unnecessary, because the main features of $C$. burnetii infection in wildlife-infection biology, transmission, pathological findings, clinical signs, shedding patterns, risk factors-do not significantly differ from what is reported in domestic ruminants (11); and (iii) useless as regards estimating the efficiency and potentiality of IPIV to control C. burnetii infection in red deer in endemic scenarios. The opportunity to test IPIV in a C. burnetii endemic red deer population was unique because access to sampling several times per year was warranted by the handling protocol. The negative aspect of targeting wildlife, although in a controlled population, is that consecutive sampling was not possible, and therefore, any short-term variation in shedding patterns associated with the vaccine may have gone unnoticed.

\section{Humoral Immune Response to Vaccination}

Vaccination and subsequent revaccination induced a high and stable humoral response in the population that remained high when the animals were revaccinated biannually. Vaccination with a single dose (first vaccination) induced a high humoral response in seronegative hinds in a short period of time (3 weeks). Although the boosting effect on the humoral immune response after revaccination could not be evaluated in the short term, the level of antibodies remained similarly high in vaccinated, previously seronegative animals a few months later. This shows 

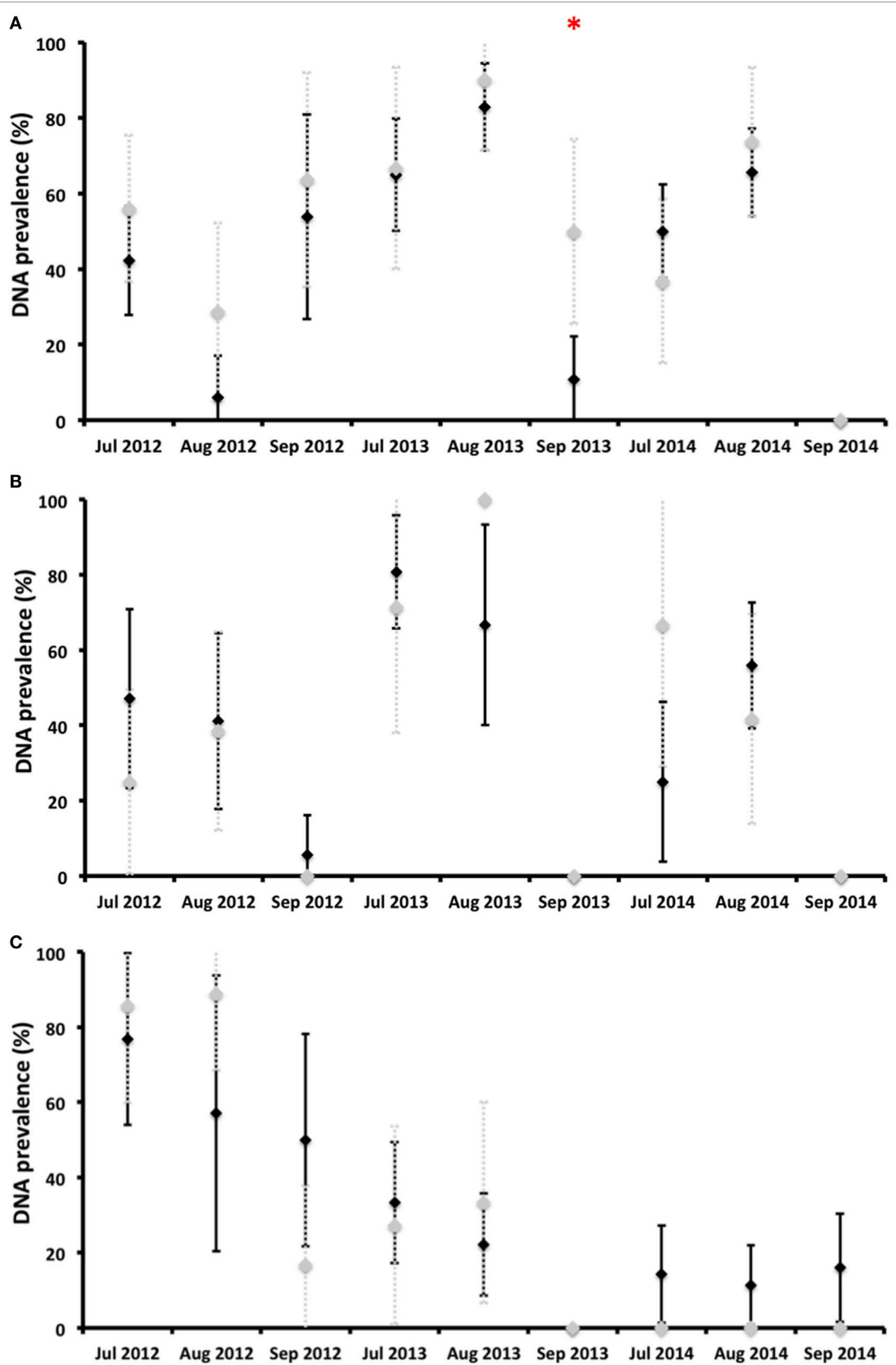

FIGURE 3 | Proportion of deer hinds (prevalence) shedding Coxiella burnetii in vaginal secretions (A), milk (B), and feces (C) in vaccinated (black diamonds) and control (gray diamonds) groups. *Statistically significant differences $(p<0.05)$ between vaccinated and control groups on a particular sampling date. 


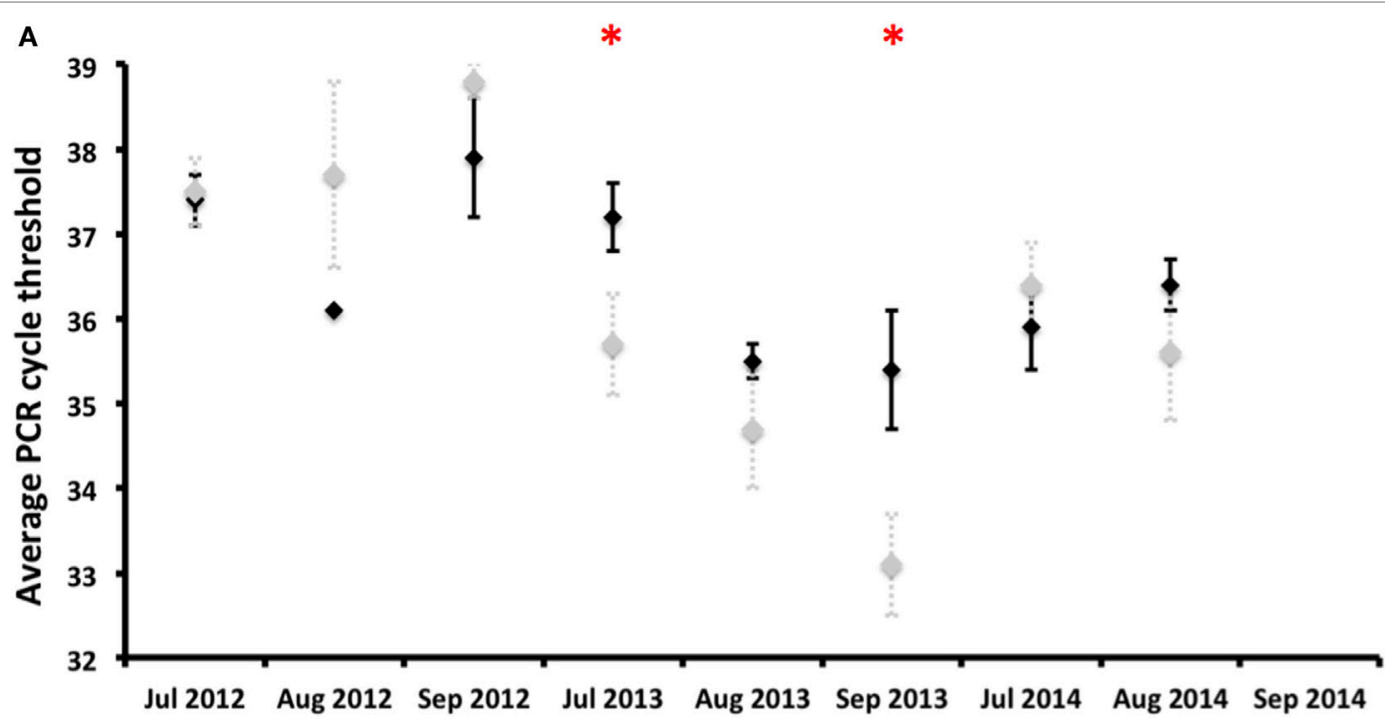

B

B

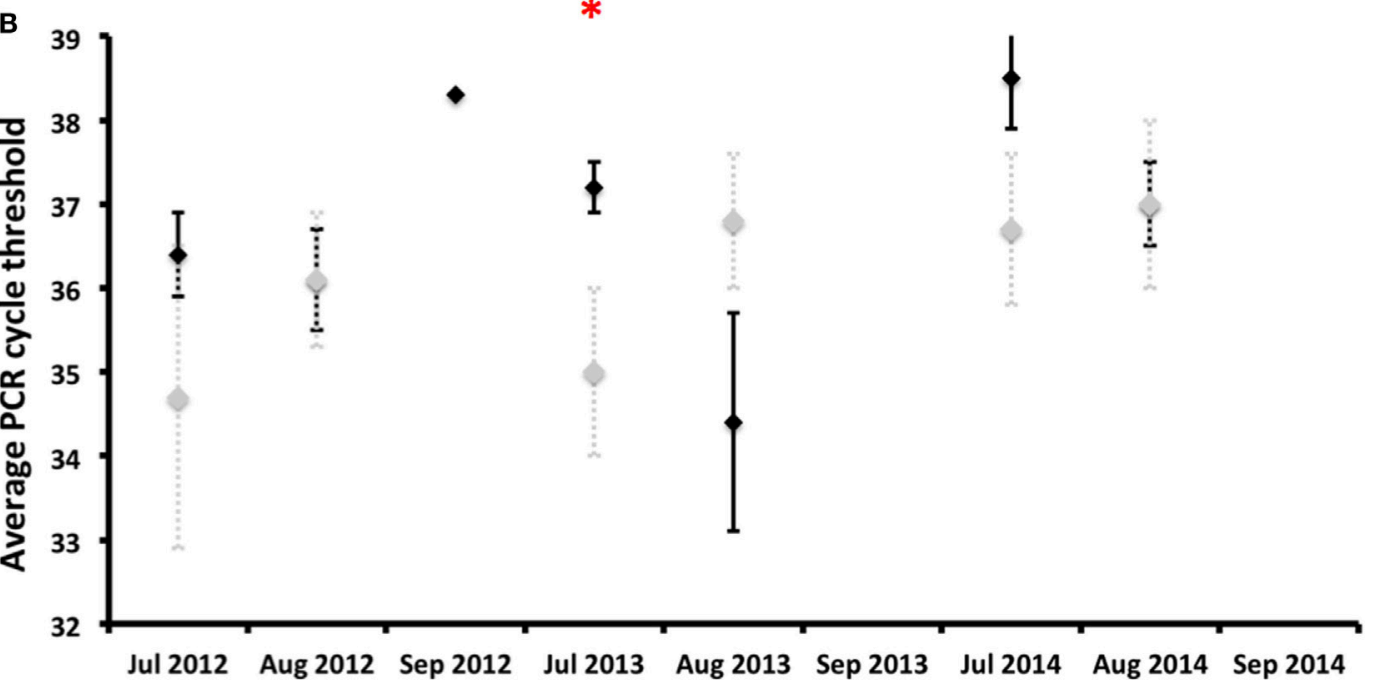

C

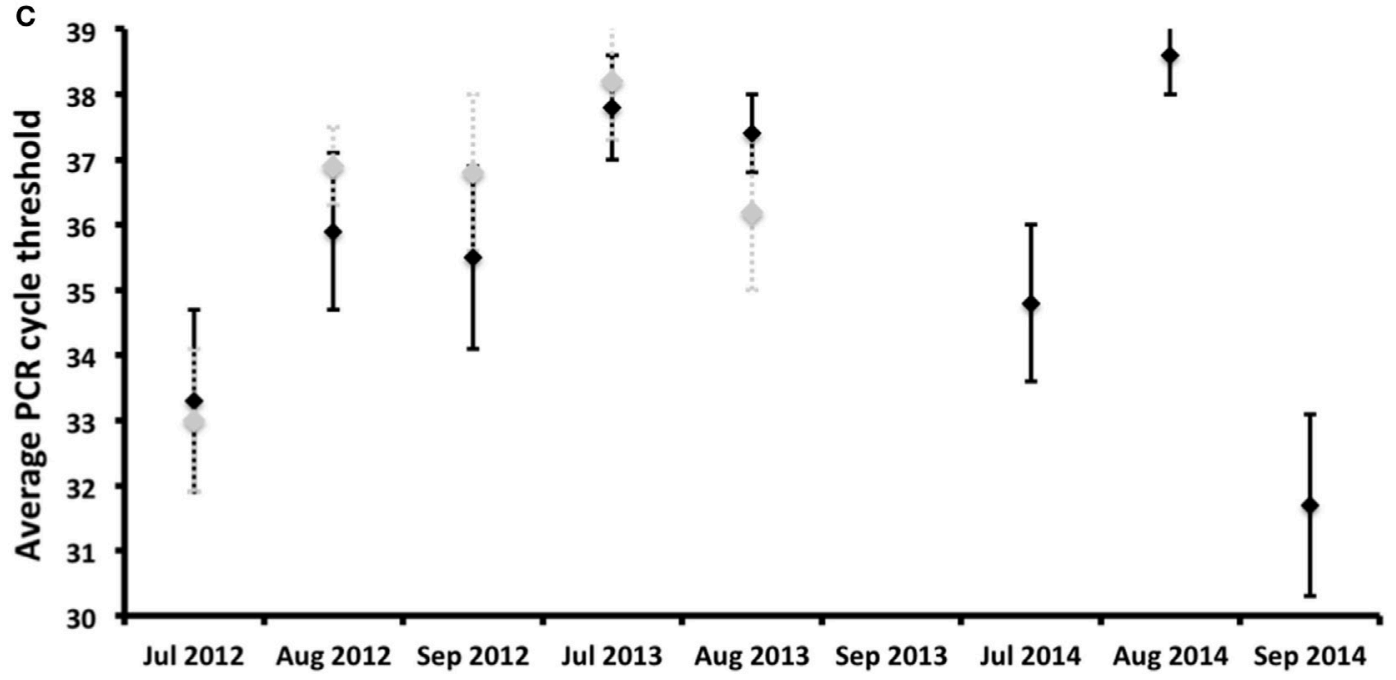

FIGURE 4 | Burden of Coxiella burnetii shed [average qPCR cycle threshold (Ct) values] by deer hinds and associated SD (error bars) as regards vaginal secretions (A), milk (B), and feces (C) displayed for vaccinated (black diamonds) and control (gray diamonds) groups. ${ }^{*}$ Statistically significant differences $(p<0.05)$ between vaccinated and control groups on a particular date. 
that revaccination every 6 months would keep high levels of antibodies circulating in vaccinated individuals. In goats, $95 \%$ seroconversion rates were observed 28 days after vaccination, and antibodies lasted 8-12 months (18). Seroconversion rates in vaccinated sheep range between 40 and $100 \%(21,36,37)$. Brooks et al. (38) detected antibodies until 11 months in sheep vaccinated with a phase I vaccine. $80 \%$ of cattle maintain antibodies 1 year after vaccination (30). In contrast, the potential vaccination failure in July 2013 (see below) showed that the average life of vaccine antibodies is shorter than in domestic ruminants.

Although we can conclude that the vaccination and revaccination of deer with Coxevac maintain high levels of anti-C. burnetii antibodies circulating, we observed a gap in the response after vaccination in July 2013; seroprevalence and antibody levels decreased by January 2014 (Figure 2). We have no explanation for this observation, but since C. burnetii antibodies in red deer last for an average of 5 to 6 months (25), this drop in the humoral response to the vaccine could be related to either the bad conservation of the vaccine or a failure of the vaccine batch employed. We assume that there were no changes in the immune capacity of the animals studied, since significant health problems were not observed in this period. Problems related to the ELISA kit batch employed were also disproved.

\section{Effects of Vaccination on C. burnetii Shedding Patterns}

Coxiella burnetii DNA was detected in vaginal swabs of vaccinated and unvaccinated hinds 4.5 months after calving, similarly to reports obtained for goats (20). Vaccination did not reduce the burden of $C$. burnetii shed in vaginal secretions throughout the study period, but the shedding time in vaginal secretions was reduced by the third year in comparison to the two previous years. A longer monitoring period would, perhaps, better show the effect of vaccination [see Ref. (29)], which was unfortunately impossible in our experiment owing to logistical and economic constraints. In vaccinated challenged goats, Arricau-Bouvery et al. (18) observed a reduction in the vaginal shedding time just 2 weeks after vaccination. Although not every vaccinated yearling deer of the 2011 and 2012 cohorts had been exposed to C. burnetii by the time of vaccination (Figure 2), we did not observe a general reduction in shedding in a short-time period. However, we observed a reduction in the vaginal shedding time, in spite of the fact that the deer were within a highly contaminated environment. We cannot, therefore, discard the hypothesis that the vaccination of unexposed deer under low infection pressure would reduce vaginal shedding in a shorter period [see Ref. (19)]. In contrast to reports obtained for goats (22), no reduction in the C. burnetii shedding burden in vaginal secretions was observed in red deer. In naturally infected sheep vaccinated for 3-4 years, the number of shedders and the vaginal bacterial burden shed also decreased only slightly with time $(21,29)$, which was interestingly more discrete than that which was observed in red deer with similar to higher individual infection rates (25).

Coxiella burnetii shedding time in milk from calving was shorter than vaginal shedding, which is consistent with the milk shedding patterns (4-6 weeks) observed in unvaccinated naturally infected goats $(18,39)$. The vaccination of goats reduced the bacterial load and time of shedding in milk (22). The shorter time of milk shedding, when compared to that of vaginal secretions or feces, coincides with previous reports concerning sheep and goats (39-41). The lack of reduction of shedding prevalence and burden coincides with some studies carried out on sheep and goats $(29,39-41)$.

Coxiella burnetii shedding in feces was detected 4.5 months after calving, as previously observed in non-vaccinated goats (39). The main finding of our study was the progressive reduction of C. burnetii shedding prevalence in feces, although not the burden of shed bacteria, over time from the implementation of the vaccination. This was observed in both the vaccinated and control groups but, since the animals in both groups were mixed in existing batches, the reduction observed in the control animals could be a consequence of the lower burden shed by vaccinated hinds. This would theoretically reduce environmental contaminationif we assume that feces are the main source of this contamination (42), which would account for the reduced infection pressure. One interesting observation as regards the study population is that the annual incidence of $C$. burnetii infection in yearlings decreased from 2012 to 2014 (25), which could perhaps support the supposition that vaccination with IPIV reduces the risk of infection by C. burnetii in red deer. Future attempts might help identify the causality relationships between fecal shedding and infection pressure.

\section{CONCLUSION}

The objective of this article is to test the efficacy of IPIV in endemic red deer populations that resemble real scenarios and estimate the usefulness of IPIV to control C. burnetii infection in free-roaming endemic red deer populations. Therefore, we followed the recommendations for endemic scenarios and used a long-term vaccination approach for the experiment. Our approach showed that IPIV could be effective in reducing C. burnetii shedding, especially in feces, if they are applied on a long-term basis (more than 3 consecutive years) and, therefore, account for a reduction in infection pressure. We vaccinated replacement hinds for the first time when they were 13 months old on the basis of preliminary data on C. burnetii infection dynamics (25), but we would recommend that future approaches target 5- to 7-month-old animals. That would perhaps protect calves from infection between the loss of maternally derived antibodies (25) and the next calving season when $C$. burnetii is shed by reproducing hinds.

\section{ETHICS STATEMENT}

The experiment was approved by the Research Ethics Commission of the Animal Ethics Committee of Castilla_La Mancha University.

\section{AUTHOR CONTRIBUTIONS}

FR-F and DG-B designed the study, analyzed the data, and wrote the manuscript; DG-B and JO collected samples; DG-B performed molecular and serological analyses; JO critically reviewed the manuscript; and all the authors approved the submitted version of the manuscript. 


\section{ACKNOWLEDGMENTS}

We would like to thank the deer keepers for their valuable help in deer handling and sample collection. This work was funded

\section{REFERENCES}

1. Maurin M, Raoult D. Q fever. Clin Microbiol Rev (1999) 12:518-53.

2. Agerholm JS. Coxiella burnetii associated reproductive disorders in domestic animals - a critical review. Acta Vet Scand (2013) 55:13. doi:10.1186/ 1751-0147-55-13

3. Georgiev M, Afonso A, Neubauer H, Needham H, Thiéry R, Rodolakis A, et al. Q fever in humans and farm animals in four European countries, 1982 to 2010. Euro Surveill (2013) 18:20407. doi:10.2807/ese.18.08.20407

4. Tempelman C, Prins J, Koopmans C. Economic Consequences of the Q-Fever Outbreak. SEO Economisch Onderzoek (2011). 15 p. Available from: http:// www.seo.nl/en/page/article/economische-gevolgen-van-de-uitbraak-van-qkoorts/.

5. Morroy G, Prins J, Bergevoet R, Schneeberger P, Bor HHJ, van der Hoek W, et al. Of goats and humans; the societal costs of the Dutch Q fever saga. Int J Infect Dis (2012) 16:e266. doi:10.1016/j.ijid.2012.05.914

6. Clemente L, Fernandes TL, Barahdna R, Bernardino R, Botelho A. Confirmation by PCR of Coxiella burnetii infection in animals at a zoo in Lisbon, Portugal. Vet Rec (2008) 163:221-2. doi:10.1136/vr.163.7.221

7. Oporto B, Barandika JF, Hurtado A, Aduriz G, Moreno B, Garcia-Perez AL. Incidence of ovine abortion by Coxiella burnetii in northern Spain. Ann N Y Acad Sci (2006) 1078:498-501. doi:10.1196/annals.1374.095

8. Lloyd C, Mark F, Stidworthy MA, Wernery U, Ulrich W. Coxiella burnetii abortion in captive dama gazelle (Gazelladama) in the United Arab Emirates. J Zoo Wildl Med (2010) 41:83-9. doi:10.1638/2009-0005.1

9. González-Barrio D, Almería S, Caro MR, Salinas J, Ortíz JA, Gortázar C, et al. Coxiella burnetii shedding by farmed red deer (Cervus elaphus). Transbound Emerg Dis (2015) 62:572-4. doi:10.1111/tbed.12179

10. Astobiza I, Barandika JF, Ruiz-Fons F, Hurtado A, Povedano I, Juste RA, et al. Coxiella burnetii shedding and environmental contamination at lambing in two highly naturally-infected dairy sheep flocks after vaccination. Res Vet Sci (2011) 91:e58-63. doi:10.1016/j.rvsc.2010.11.014

11. González-Barrio D, Vieira-Pinto M, Ruiz-Fons F. Coxiella burnetii in European game species: challenges for human health. In: Simoes JCC, Anastácio SF, Da Silva GJ, editors. The Principles and Practice of Q Fever. The One Health Paradigm. New York: Nova Science Publishers (2017). p. 245-72.

12. Gortázar C, Reperant LA, Kuiken T, de la Fuente J, Boadella M, MartínezLópez B, et al. Crossing the interspecies barrier: opening the door to zoonotic pathogens. PLoS Path (2014) 10:e1004129. doi:10.1371/journal.ppat.1004129

13. Ruiz-Fons F. A review of the current status of relevant zoonotic pathogens in wild swine (Sus scrofa) populations: changes modulating the risk of transmission to humans. Tranbound Emerg Dis (2017) 64:68-88. doi:10.1111/ tbed.12369

14. Apollonio M, Andersen R, Putman R. European Ungulates and Their Management in the 21st Century. Cambridge: Cambridge University Press (2010). $604 \mathrm{p}$.

15. González-Barrio D, Velasco Ávila AL, Boadella M, Beltrán-Beck B, Barasona JA, Santos JPV, et al. Host and environmental factors modulate the exposure of free-ranging and farmed red deer (Cervus elaphus) to Coxiella burnetii. Appl Environ Microbiol (2015) 81:6223-31. doi:10.1128/AEM.01433-15

16. Ruiz-Fons F, Astobiza I, Barandika JF, Hurtado A, Atxaerandio R, Juste RA, et al. Seroepidemiological study of $\mathrm{Q}$ fever in domestic ruminants in semiextensive grazing systems. BMC Vet Res (2010) 6:3. doi:10.1186/1746-6148-6-3

17. González-Barrio D, Hagen F, Tilburg JJHC, Ruiz-Fons F. Coxiella burnetii genotypes in Iberian wildlife. Microb Ecol (2016) 72:890-7. doi:10.1007/ s00248-016-0786-9

18. Arricau-Bouvery N, Souriau A, Bodier C, Dufour P, Rousset E, Rodolakis A. Effect of vaccination with phase I and phase II Coxiella burnetii vaccines in pregnant goats. Vaccine (2005) 23:4392-402. doi:10.1016/j.vaccine.2005.04.010

19. Guatteo R, Seegers H, Joly A, Beaudeau F. Prevention of Coxiella burnetii shedding in infected dairy herds using a phase I C. burnetii inactivated vaccine. Vaccine (2008) 26:4320-8. doi:10.1016/j.vaccine.2008.06.023 by EU FP7 Grant ANTIGONE (278976) and CDTI (Centro para el Desarrollo Tecnológico Industrial, Spanish Ministry for the Economy and Competitiveness-MINECO). FR-F is supported by a "Ramón y Cajal" contract from MINECO.

20. Rousset E, Durand B, Champion JL, Prigent M, Dufour P, Forfait C, et al. Efficiency of a phase 1 vaccine for the reduction of vaginal Coxiella burnetii shedding in a clinically affected goat herd. Clin Microbiol Infect (2009) 15: 188-9. doi:10.1111/j.1469-0691.2008.02220.x

21. Astobiza I, Barandika JF, Ruiz-Fons F, Hurtado A, Povedano I, Juste RA, et al. Four-year evaluation of the effect of vaccination against Coxiella burnetii on reduction of animal infection and environmental contamination in a naturally infected dairy sheep flock. Appl Environ Microbiol (2011) 77:7405-7. doi:10.1128/AEM.05530-11

22. Hogerwerf L, van den Brom R, Roest HI, Bouma A, Vellema P, Pieterse M, et al. Reduction of Coxiella burnetii prevalence by vaccination of goats and sheep, the Netherlands. Emerg Infect Dis (2011) 17:379-86. doi:10.3201/eid1703.101157

23. Angelakis E, Raoult D. Q fever. Vet Microbiol (2010) 140:297-309. doi:10.1016/ j.vetmic.2009.07.016

24. Ruiz-Fons F, Rodríguez O, Torina A, Naranjo V, Gortázar C, de la Fuente J. Prevalence of Coxiella burnetii infection in wild and farmed ungulates. Vet Microbiol (2008) 126:282-6. doi:10.1016/j.vetmic.2007.06.020

25. González-Barrio D, Fernández-de-Mera IG, Ortiz JA, Queirós J, Ruiz-Fons F. Long-term dynamics of Coxiella burnetii in farmed red deer (Cervus elaphus). Front Vet Sci (2015) 2:74. doi:10.3389/fvets.2015.00074

26. Kreizinger Z, Szeredi L, Bacsadi A, Nemes C, Sugár L, Varga T, et al. Occurrence of Coxiella burnetii and Chlamydiales species in abortions of domestic ruminants and in wild ruminants in Hungary, Central Europe. J Vet Diagn Invest (2015) 27:206-10. doi:10.1177/1040638714563566

27. González-Barrio D, Maio E, Vieira-Pinto M, Ruiz-Fons F. European rabbits as reservoir for Coxiella burnetii. Emerg Infect Dis (2015) 21:1055-8. doi:10.3201/ eid2106.141537

28. González-Barrio D, Jado I, Fernández-de-Mera IG, Fernández-Santos MR, Rodríguez-Vargas M, García-Amil C, et al. Genotypes of Coxiella burnetii in wildlife: disentangling the molecular epidemiology of a multi-host pathogen. Environ Microbiol Rep (2016) 8:708-14. doi:10.1111/1758-2229. 12431

29. Astobiza I, Barandika JF, Juste RA, Hurtado A, García-Pérez AL. Evaluation of the efficacy of oxytetracycline treatment followed by vaccination against $Q$ fever in a highly infected sheep flock. Vet J (2013) 196:81-5. doi:10.1016/j.tvjl.2012.07.028

30. Rodolakis A, Clement P, Cochonneau D, Beaudeau F, Sarradin P, Guatteo R. Investigation of humoral and cellular immunity of dairy cattle after one or two year of vaccination with a phase I Coxiella vaccine. Procedia Vaccinol (2009) 1:85-8. doi:10.1016/j.provac.2009.07.015

31. Tilburg JJHC, Melchers WJ, Petterson AM, Rossen JM, Hermans MH, van Hannen EJ, et al. Interlaboratory evaluation of different extraction and realtime PCR methods for detection of Coxiella burnetii DNA in serum. J Clin Microbiol (2010) 48:3923-7. doi:10.1128/JCM.01006-10

32. Kaden V, Lange E, Steyer H. Does multiple oral vaccination of wild boar against classical swine fever (CSF) have a positive influence on the immunity? Dtsch Tierarztl Wochenschr (2004) 111:63-7.

33. Garrido JM, Sevilla IA, Beltrán-Beck B, Minguijón E, Ballesteros C, Galindo RC, et al. Protection against tuberculosis in Eurasian wild boar vaccinated with heat-inactivated Mycobacterium bovis. PLoS One (2011) 6:e24905. doi:10.1371/ journal.pone.0024905

34. Freuling CM, Hampson $\mathrm{K}$, Selhorst T, Schröder R, Meslin FX, Mettenleiter TC, et al. The elimination of fox rabies from Europe: determinants of success and lessons for the future. Philos Trans R Soc Lond B Biol Sci (2013) 368:20120142. doi:10.1098/rstb.2012.0142

35. Ballesteros C, Vicente J, Carrasco-García R, Mateo R, de la Fuente J Gortázar C. Specificity and success of oral-bait delivery to Eurasian wild boar in Mediterranean woodland habitats. Eur J Wildl Res (2011) 57:749-57. doi:10.1007/s10344-010-0483-9

36. Eibach R, Bothe F, Runge M, Ganter M. Long-term monitoring of a Coxiella burnetii-infected sheep flock after vaccination and antibiotic treatment under field conditions. Berl Munch Tierarztl Wochenschr (2013) 126:3-9. doi:10.2376/0005-9366-126-3 
37. Hamann HP, Volmer R, Wimmershof N, Ballmann G. Zschöck M.Q-fevervaccination in sheep. Tierarztl Umsch (2009) 64:188-90.

38. Brooks DL, Ermel RW, Franti CE, Ruppanner R, Behymer DE, Williams JC, et al. Q fever vaccination of sheep: challenge of immunity in ewes. Am J Vet Res (1986) 47:1235-8.

39. Roest HJ, van Gelderen B, Dinkla A, Frangoulidis D, van Zijderveld F, Rebel J, et al. Q Fever in pregnant goats: pathogenesis and excretion of Coxiella burnetii. PLoS One (2012) 7:e48949. doi:10.1371/journal.pone.0048949

40. Rodolakis A, Berri M, Héchard C, Caudron C, Souriau A, Bodier CC, et al. Comparison of Coxiella burnetii shedding in milk of dairy bovine, caprine, and ovine herds. J Dairy Sci (2007) 90:5352-60. doi:10.3168/jds.2006-815

41. Astobiza I, Barandika JF, Hurtado A, Juste RA, Garcia-Perez AL. Kinetics of Coxiella burnetii excretion in a commercial dairy sheep flock after treatment with oxytetracycline. Vet $J$ (2010) 184:172-5. doi:10.1016/j.tvjl.2009. 01.017
42. Courcoul A, Monod H, Nielen M, Klinkenberg D, Hogerwerf L, Beaudeau F, et al. Modelling the effect of heterogeneity of shedding on the within herd Coxiella burnetii spread and identification of key parameters by sensitivity analysis. J Theor Biol (2011) 284:130-41. doi:10.1016/j.jtbi.2011.06.017

Conflict of Interest Statement: The authors declare that the research was conducted in the absence of any commercial or financial relationships that could be construed as a potential conflict of interest.

Copyright (๑) 2017 González-Barrio, Ortiz and Ruiz-Fons. This is an open-access article distributed under the terms of the Creative Commons Attribution License (CC BY). The use, distribution or reproduction in other forums is permitted, provided the original author(s) or licensor are credited and that the original publication in this journal is cited, in accordance with accepted academic practice. No use, distribution or reproduction is permitted which does not comply with these terms. 